\title{
Recursive Algorithms for the Matrix Padé Problem
}

\author{
By Adhemar Bultheel
}

\begin{abstract}
A matrix triangularization interpretation is given for the recursive algorithms computing the Padé approximants along a certain path in the Padé table, which makes it possible to unify all known algorithms in this field [5], [6]. For the normal Padé table, all these results carry over to the matrix Pade problem in a straightforward way. Additional features, resulting from the noncommutativity are investigated. A generalization of the Trench-Zohar algorithm and related recursions are studied in greater detail.
\end{abstract}

1. Introduction. Let $K[z]$ be the set of formal power series over a ring $K$ with indeterminate $z$. For ease of exposition, we restrict $K$ to be the ring of $n \times n$ matrices, though a more general setting is possible [3].

Let $d^{\circ} P(z)$ denote the degree of a matrix polynomial $P(z)$ and ord $Z(z)$ the order of a formal power series $Z(z)$, i.e.

$$
d^{\circ} P(z)=N \Leftrightarrow P(z)=\sum_{k=0}^{N} p_{k} z^{k}, \quad p_{N} \neq 0, p_{k} \in K, k=0,1, \ldots, N,
$$

and

ord $Z(z)=M \Leftrightarrow Z(z)=\sum_{k=M}^{\infty} r_{k} z^{k}, \quad r_{M} \neq 0, r_{k} \in K, k=M, M+1, \ldots$

For given nonnegative integers $M$ and $N$, and some $F(z) \in K[z]$, the right Padé approximation problem consists in finding two matrix polynomials $P^{[M / N]}(z)$ and $Q^{[M / N]}(z)$ such that
(a) $d^{\circ} P^{[M / N]}$
$(z) \leqslant M, d^{\circ} Q^{[M / N]}(z) \leqslant N$
(b) $P^{[M / N]}(z)$ and $Q^{[M / N]}(z)$ have no common right divisor of degree $>0$,
(c) the right $(R-)$ residual $Z^{[M / N]}(z)$, defined by

$$
Z^{[M / N]}(z)=F(z) Q^{[M / N]}(z)-P^{[M / N]}(z)
$$

satisfies

$$
\text { ord } Z^{[M / N]}(z) \geqslant M+N+1 \text {, and }
$$

(d) the constant term $q_{0}^{[M / N]}$ of $Q^{[M / N]}(z)$ equals $I$.

Received May 11, 1979; revised September 24, 1979.

1980 Mathematics Subject Classification. Primary 41A20, 65F05.

Key words and phrases. Hankel and Toeplitz matrices, triangular decomposition of matrices, fast algorithms, rational approximation. 
$\left(P^{[M / N]}(z)\right)\left(Q^{[M / N]}(z)\right)^{-1}$ is denoted by $A^{[M / N]}(z)$ and is called the $(M, N)$ right Padé approximant (RPA) of $F(z)$. Such RPA's need not exist for all $M, N \geqslant 0$; but for ease in exposition, we suppose they all do.

Suppose $F(z)=\Sigma_{k=0}^{\infty} f_{k} z^{k}$ is given and that for two integers $M$ and $N$ we have the following expansions of $R$-numerator, $R$-denominator and $R$-residual of the $(M, N)$ RPA of $F(z)$

$$
\begin{gathered}
P^{[M / N]}(z)=\sum_{k=0}^{M} p_{k}^{[M / N]} z^{k}, \quad Q^{[M / N]}(z)=\sum_{k=0}^{N} q_{k}^{[M / N]} z^{k}, \quad \text { and } \\
Z^{[M / N]}(z)=\sum_{k=0}^{\infty} r_{k}^{[M / N]} z^{M+N+1+k}
\end{gathered}
$$

Requirement (c) boils down to the following block system of equations:

$$
\left[\begin{array}{c}
T^{[M / N]} \\
-\bar{T}-\overline{1} \\
T_{0}^{[M / N]} \\
T_{+}^{[M / N]}
\end{array}\right] Q^{[M / N]}=\left[\begin{array}{c}
P^{[M / N]} \\
---- \\
0 \\
----- \\
0
\end{array}\right]+Z^{[M / N]} \quad \text { with } Z^{[M / N]}=\left[\begin{array}{c}
0 \\
---- \\
-\frac{0}{\left.R^{[M / N}\right]}
\end{array}\right]
$$

which is a shorthand notation for

(1)

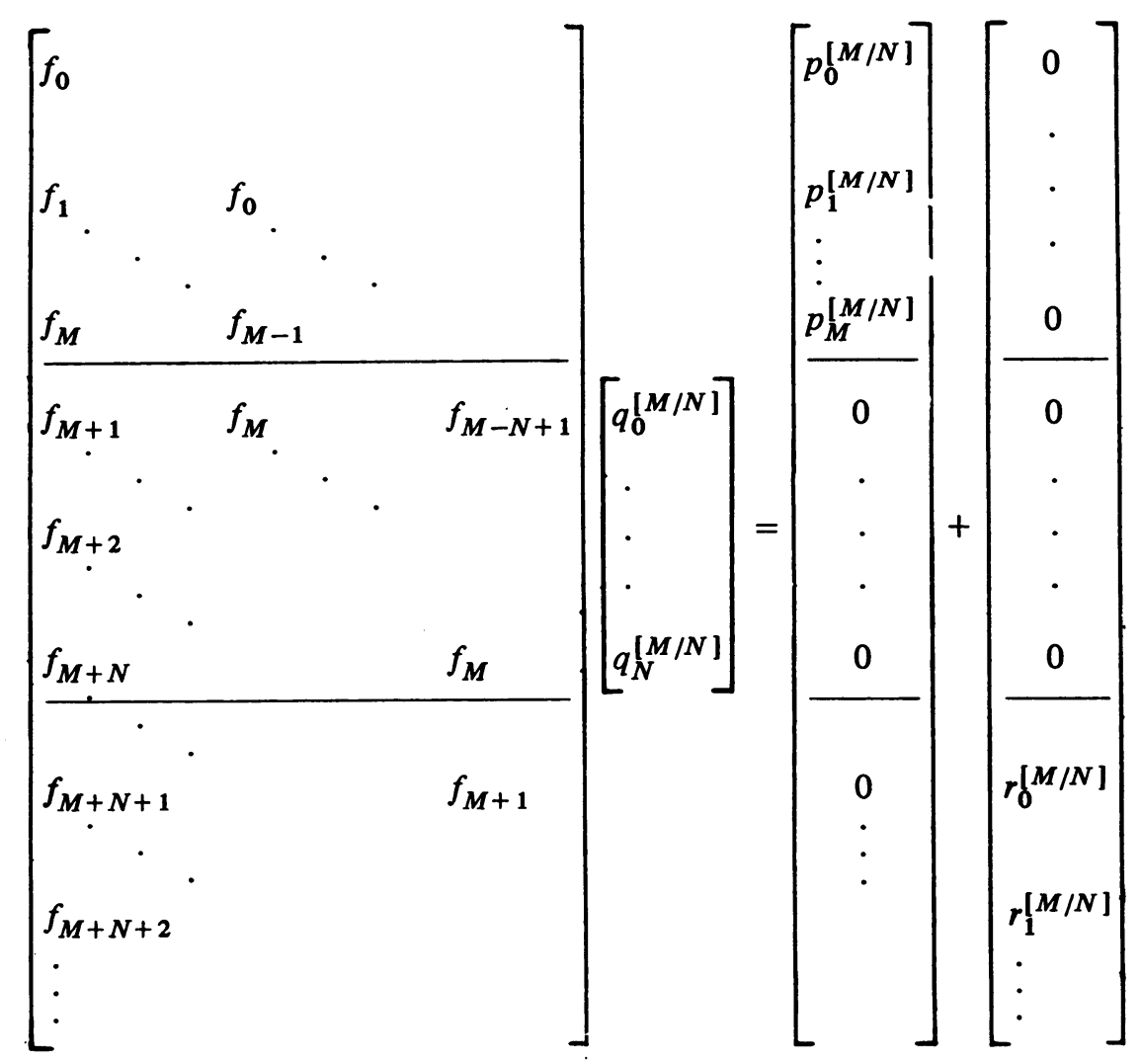


The $R$-numerator coefficients in $P^{[M / N]}$ and the $R$-residual coefficients in $R^{[M / N]}$ may be recovered from $P^{[M / N]}=T^{[M / N]} Q^{[M / N]}$ and $R^{[M / N]}=$ $T_{+}^{[M / N]} Q^{[M / N]}$, once $Q^{[M / N]}$ is known. The denominator coefficients must satisfy

$$
q_{0}^{[M / N]}=I \text { and } T_{0}^{[M / N]} Q^{[M / N]}=0,
$$

where $T_{0}^{[M / N]}$ has dimension $N n \times(N+1) n$ and 0 has dimension $N n \times n$. Equivalent forms of this set of equations are

$$
\begin{aligned}
& T^{[M / N-1]}\left[q_{1}^{[M / N]} \cdots q_{N}^{[M / N]}\right]^{t}=-\left[f_{M+1} \cdots f_{M+N}\right]^{t}, \\
& T^{[M+1 / N]} Q^{[M / N]}=\left[0 \cdots 0 r_{0}^{[M / N]}\right]^{t}, \quad q_{0}^{[M / N]}=I,
\end{aligned}
$$

and

$$
T^{[M / N]} Q^{[M / N]}=\left[p_{M}^{[M / N]} 0 \cdots 0\right]^{t}, \quad q_{0}^{[M / N]}=I,
$$

where the superscript $t$ denotes the block transpose of a block matrix and

$$
T^{[I / J]}=\left[\begin{array}{ccc}
f_{I} & \cdots & f_{I-J} \\
\vdots & \ddots & \vdots \\
f_{I+J} & \cdots & f_{I}
\end{array}\right] \text { for } I, J \geqslant 0 .
$$

(A negatively indexed $f$ must be taken as zero.)

Equation (2a) has a solution if

$$
T^{[M / N-1]} \text { and }\left[T^{[M / N-1]} \mid \begin{array}{c}
f_{M+1} \\
\vdots \\
f_{M+N}
\end{array}\right]
$$

have the same rank. Clearly, det $T^{[M / N-1]} \neq 0$ is a sufficient, but not a necessary, condition for this to happen. However, to avoid any possible difficulty, let us suppose that $F(z)$ is such that $\operatorname{det} T^{[M / N]} \neq 0$ for all $M, N \geqslant 0$, which we call the $R$-normality condition. It implies e.g. that $f_{0}$ is nonsingular, so that the inverse formal power series $F(z)^{-1}$ exists. It also implies that $q_{N}^{[M / N]}, p_{M}^{[M / N]}, p_{0}^{[M / N]}$ and $r_{0}^{[M / N]}$ are all nonsingular for $M$ and $N \geqslant 0$. Consequently, we could, instead of the normalizing condition $q_{0}^{[M / N]}=I$ in (d), also consider a monic normalization for the $R$-denominator or a monic or comonic normalization for the $R$-numerator. Indeed, the purpose of (d) is to guarantee that $\left(Q^{[M / N]}(z)\right)^{-1}$ exists (therefore, $q_{0}^{[M / N]}$ should be nonsingular) and to pin down the arbitrary constant right factor that remains as a degree of freedom in the $R$-numerator and $R$-denominator if only (a), (b) and (c) were imposed. From now on we use as a normalizing condition either (d1) $q_{0}^{[M / N]}=I$ (comonic normalization) or (d2) $q_{N}^{[M / N]}=I$ (monic normalization).

The foregoing can be adapted for a left Padé approximant (LPA). If we use the same notations as for the RPA, but transfer the superscript $[M / N]$ from right to left, then the LPA equals e.g. ${ }^{[M / N]} A(z)=\left({ }^{[M / N]} Q(z)\right)^{-1}\left({ }^{[M / N]} P(z)\right)$ and the $L$-numerator ${ }^{[M / N]} P(z)$ and the $L$-denominator ${ }^{[M / N]} Q(z)$ must satisfy 


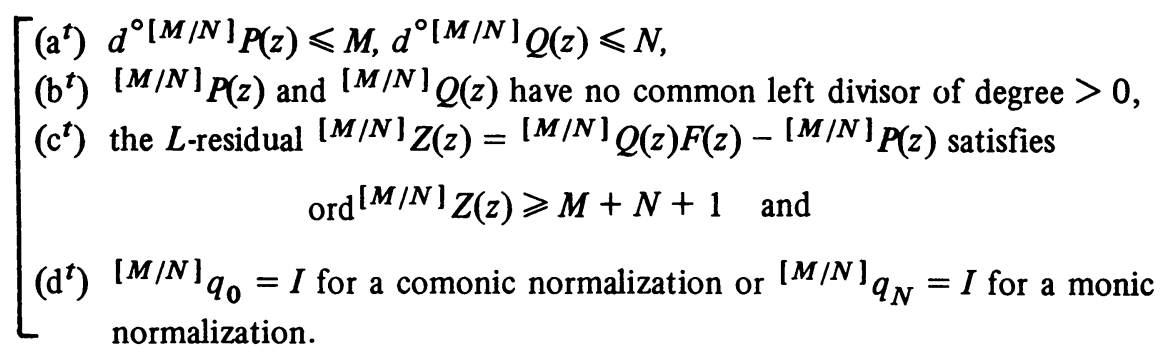

The analog of system (2a) is

$$
\left[^{[M / N]} q_{1} \cdots[M / N] q_{N}\right]^{[M / N-1]} T=-\left[f_{M+1} \cdots f_{M+N}\right]
$$

with ${ }^{[M / N]} T^{t}=T^{[M / N]}$. The block systems (2a) and $\left(2 \mathrm{a}^{t}\right)$ are essentially different since $(B C)^{t} \neq C^{t} B^{t}$ for general block matrices $B$ and $C$.

Some further notational conventions and definitions are resumed. $J$ denotes the block permutation matrix

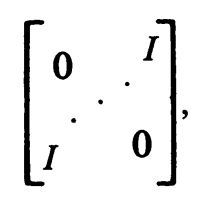

with $I$ the $n \times n$ identity matrix and $J$ of appropriate dimensions.

For a block column vector $V, \hat{V}$ is the block reciprocal vector: $\hat{V}=J V$.

[ø] denotes an empty matrix, i.e. matrix of dimension $n \times 0,0 \times n$ or $0 \times 0$ as will be appropriate.

If $S(z)$ is a formal power series or a polynomial, then by $S$ (without an argument) we mean the corresponding block column vector of its coefficients.

$e_{j}, j=0,1,2, \ldots$, are the block unit vectors. $e_{j}$ is a block column vector of an appropriate number of $0_{n \times n}$ blocks, except for the $j$ th block row, which is $I_{n \times n}$.

The chopping operators $\neg$ and $\lrcorner$ are defined as

$$
\left.\neg=\left[0 \mid e_{1} e_{2} \cdots e_{N}\right] \text { and }\right\lrcorner=\left[e_{1} e_{2} \cdots e_{N} \mid 0\right] .
$$

A square block matrix $W$ is called block persymmetric if $J W^{t} J=W$. A block Toeplitz matrix is an example of a block persymmetric matrix. Remark that although, in general, there is no relation between the rank of a block matrix $W$ and the rank of $W^{t}$, it is clear that for a block persymmetric matrix $T, T^{t}=J T J$ and $T$ have the same rank. Consequently, the $R$-normality condition coincides with its $L$-dual version.

It takes little effort to prove that ${ }^{[M / N]} A(z)=A^{[M / N]}$ (z) (see e.g. [3]), so that we can use $[M / N]$ to denote the $(M, N)$ Padé approximant $(\mathrm{PA})$ without $L$ - or $R$-specification. If the normality condition is satisfied, then all the PA's $[M / N]$, $M, N \geqslant 0$, exist and they can be arranged in a matrix having $[M / N]$ at the intersection of row $M$ and column $N$. This matrix is called the Padé table of $F(z)$. Under the above conditions $F(z)$ and its Padé table are called normal.

We will give algorithms to find the solutions of $(2)$ or $\left(2^{t}\right)$ or of related problems when the indices $M$ and $N$ are varying such that the PA's $[M / N]$ make up certain paths in the Pade table. There are $L$ - and $R$-versions of the algorithms, depending on whether the $L$ - or $R$-numerators and denominators of the $[M / N]$ are computed. 
We use also the notions of right and left continued fractions (RCF and LCF). A RCF is denoted by

$$
a_{0}+\frac{\left.a_{1}\right\rfloor}{\left\lceil b_{1}\right.}+\frac{\left.a_{2}\right\rfloor}{\left\lceil b_{2}\right.}+\cdots=a_{0}+\sum_{k=1}^{\infty} \frac{\left.a_{k}\right\rfloor}{\left\lceil b_{k}\right.}
$$

and we mean by it the (ordered) set of convergents $\left\{c_{n}\right\}_{0}^{\infty}$ with $c_{n}=P_{n} Q_{n}^{-1}, n \geqslant 0$, and

$$
\begin{array}{llll}
P_{-1}=I, & P_{0}=a_{0}, & P_{i+1}=P_{i-1} a_{i+1}+P_{i} b_{i+1} & \text { for } i \geqslant 0, \\
Q_{-1}=0, & Q_{0}=I, & Q_{i+1}=Q_{i-1} a_{i+1}+Q_{i} b_{i+1} & \text { for } i \geqslant 0 .
\end{array}
$$

A LCF is denoted by

$$
\cdots+\frac{\mid{ }_{2} a}{{ }_{2} b}+\frac{{ }_{1} a}{{ }_{1} b}\left|+{ }_{0} a=\sum_{k=1}^{\infty} \frac{\mid k_{k} a}{b}\right|+{ }_{0} a
$$

with convergents $\left\{{ }_{n} c={ }_{n} Q^{-1}{ }_{n} P\right\}_{0}^{\infty}$, where

$$
\begin{aligned}
& { }_{-1} P=I, \quad{ }_{0} P={ }_{0} a, \quad{ }_{i+1} P={ }_{i+1} a_{i-1} P+{ }_{i+1} b_{i} P \quad \text { for } i \geqslant 0, \\
& { }_{-1} Q=0, \quad{ }_{0} Q=I, \quad{ }_{i+1} Q={ }_{i+1} a_{i-1} Q+{ }_{i+1} b_{i} Q \quad \text { for } i \geqslant 0 .
\end{aligned}
$$

Unless stated otherwise, the nomenclature will have to be understood in block sense wherever appropriate, e.g. a row will refer to a block row, triangular will mean block triangular, etc.

2. Paradiagonals. Suppose we want to compute the $k$ th paradiagonal of the Padé table, defined by

$$
\begin{aligned}
& D_{k}=\{[k+j / j]\}_{j=0}^{\infty} \quad \text { if } k \geqslant 0 \quad \text { and by } \\
& D_{k}=\{[j /-k+j]\}_{j=0}^{\infty} \quad \text { if } k<0 .
\end{aligned}
$$

Let us start with $k \geqslant 0$. Take the systems $(2 \mathrm{~b})$ with a column permutation, then we have to solve recursively for $j=0,1, \ldots$,

$$
\left(T^{[k+j+1 / j]} J\right)\left(J Q^{[k+j / j]}\right)=e_{j} r^{[k+j / j]} \equiv H_{j} Y_{j}=e_{j} r_{j}
$$

Here and in the following, the right-hand side (RHS) of $\equiv$ will be an abbreviation for the left-hand side (LHS) where obvious identifications must be made.

The matrices $H_{j}$ are Hankel matrices; and if we rename $f_{k+1+j}$ as $h_{j}, j=$ $0,1, \ldots$, then we have the following nesting property for the family $H_{j}$

$$
H_{j+1}=\left[\begin{array}{c|c}
H_{j} & V_{j} \\
\hline V_{j}^{t} & h_{2 j+2}
\end{array}\right] \text { with } H_{0}=h_{0}, V_{j}^{t}=\left[h_{j+1} \cdots h_{2 j+1}\right] .
$$

The recursive solution of the family of Hankel systems (3) can be found as in the scalar case (see [5], [6] for details), and you have that for $j=0,1,2, \ldots$, 


$$
Y_{j+1}=\left[\begin{array}{c}
0 \\
Y_{j}
\end{array}\right]-\left[\begin{array}{c}
Y_{j-1} \\
0 \\
0
\end{array}\right] r_{j-1}^{-1} r_{j}-\left[\begin{array}{c}
Y_{j} \\
0
\end{array}\right] r_{j}^{-1}\left(r_{j}^{\prime}-r_{j-1}^{\prime} r_{j-1}^{-1} r_{j}\right),
$$

with

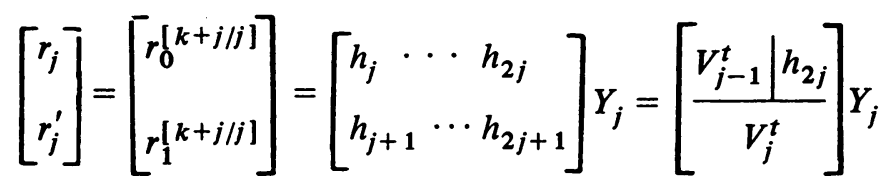

and initial conditions

$$
Y_{0}=I, \quad Y_{-1}=[\varnothing], \quad r_{0}=h_{0}, \quad r_{0}^{\prime}=h_{1}, \quad r_{-1}=I, \quad r_{-1}^{\prime}=0 .
$$

This corresponds to the following triangularization of $H_{N}$

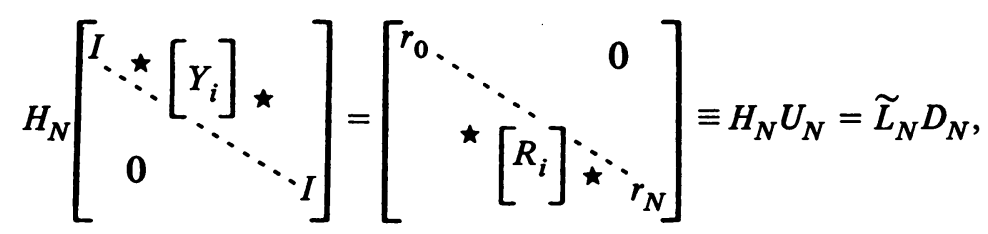

with $D_{N}=\operatorname{diag}\left(r_{0}, r_{1}, \ldots, r_{N}\right)$ and $\widetilde{L}_{N}$ unit lower triangular. $Y_{i}=\hat{Q}^{[k+i / i]}$ by definition, and from (1) it can be seen that $R_{i}$ consists of the first nonzero blocks of $Z^{[k+i / i]}$, i.e. the first $N-i+1$ nonzero $R$-residual coefficients of $[k+i / i]$. It is not difficult to verify that the $L$-denominator of the PA can be found from a similar scheme. If we denote by ${ }_{i} Y$ the reciprocal $L$-denominator vector ${ }_{i} Y={ }^{[k+i / i]} \hat{Q}$ and by ${ }_{i} R$ the first $N-i+1$ nonzero rows in the corresponding $L$-residual vector ${ }^{[k+i / i]} Z$, then

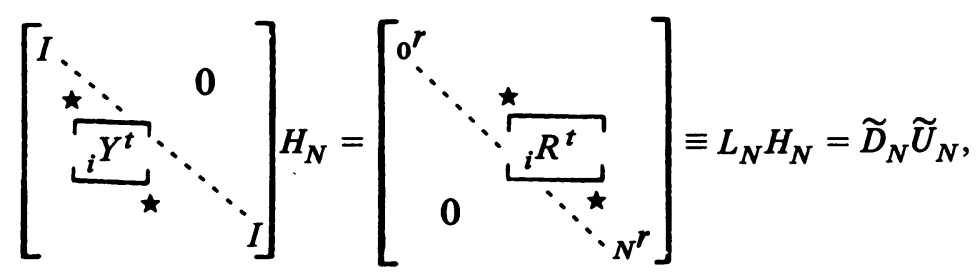

with $\widetilde{D}_{N}=\operatorname{diag}\left({ }_{0} r,{ }_{1} r, \ldots,{ }_{N} r\right)$ and $\widetilde{U}_{N}$ unit upper triangular. The factorization $\left(5^{t}\right)$ corresponds to the one proposed by Rissanen [13].

Notice that

$$
L_{N} H_{N} U_{N}=D_{N}=\widetilde{D}_{N}
$$

thus that

$$
H_{N}^{-1}=U_{N} D_{N}^{-1} L_{N} \quad \text { and } \quad H_{N}=\widetilde{L}_{N} \widetilde{D}_{N} \widetilde{U}_{N}
$$

and also

$$
\widetilde{U}_{N}=U_{N}^{-1} \text { and } \quad \widetilde{L}_{N}=L_{N}^{-1}
$$


But now, unlike in the scalar case, $U_{N}^{t} \neq L_{N}$ in general.

The above result can be summarized as follows: The $L$ - and $R$-denominators make up the triangular factors of $H_{N}^{-1}$, while part of the corresponding residuals make up the triangular factors of $H_{N}$ itself. Notice also that (6) expresses the biorthogonality of the reversed $L$ - and $R$-denominators.

Besides the $L D U$ factorization of $H_{N}$, given in (5) and $\left(5^{t}\right)$, we could also look for a $U D L$ factorization of this Hankel matrix. The triangular factors that appear then are not nested as are the triangulars in (5). All the nonzero elements depend upon $N$. Suppose we have

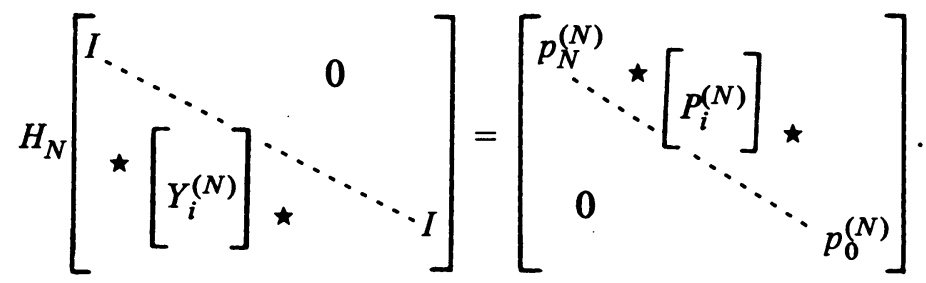

By taking the first column in (8), one obtains a system like (2c). From this, it can be seen that $Y_{N}^{(N)}$ is the reciprocal $R$-denominator of the PA $[k+N+1 / N]$ with a monic normalization and $P_{N}^{(N)}=\left[p_{N}^{(N)}\right]$ is the coefficient of highest degree in the corresponding $R$-numerator. This proves part of the more general result that relates all the columns $Y_{i}^{(N)}$ and $P_{i}^{(N)}, i=0,1, \ldots, N$, in a similar way to the PA's in the antidiagonal $\{[k+2 N+1-j / j]\}_{j=0}^{N}$. This will be explained further in Section 4 .

The paradiagonal $D_{k}$ for $k<0$ can be computed in two ways. Either we first compute the inverse formal power series $F(z)^{-1}$, and then the recursion (4) can be used unaltered or we change the initial conditions of (4) and use the $F(z)$ series again. Let us explain both possibilities.

If $[M / N]$ is the $(M, N)$ PA of $F(z)$ with $R$-denominator $Q^{[M / N]}(z)$ and $R$ numerator $P^{[M / N]}(z)$, then from the definitions it follows that, up to some normalization, $Q^{[M / N]}(z)$ is the $R$-numerator and $P^{[M / N]}(z)$ is the $R$-denominator of the $(N, M)$ PA of $F(z)^{-1}$. The $R$-denominator recursion in (4) when applied to the $F(z)^{-1}$ series is actually an $R$-numerator recursion for the $F(z)$ series.

The other possibility is to let the derivation of the algorithm for $k \geqslant 0$ go through, except that $[k+j / j]$ as an index is replaced by $[j /-k+j]$ and the initializations are adapted correspondingly. The latter have to be

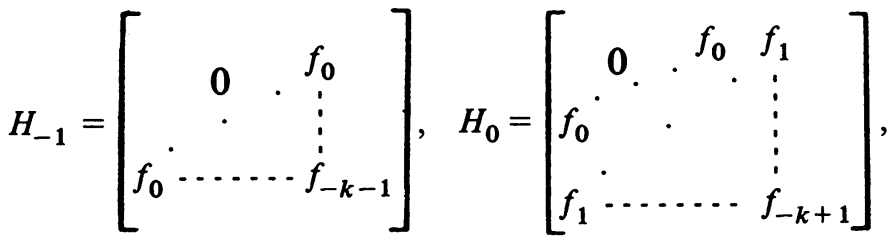

$$
\begin{aligned}
& Y_{-1}=[\underbrace{0 \cdots 0}_{-k-1}]^{t}, \quad r_{-1}=f_{0}=h_{-k-1}, \quad r_{-1}^{\prime}=f_{1}=h_{-k} \text {, } \\
& r_{0}=\left[f_{1} \cdots f_{-k+1}\right] Y_{0}, \quad r_{0}^{\prime}=\left[f_{2} \cdots f_{-k+2}\right] Y_{0},
\end{aligned}
$$


and $Y_{0}$ is the solution of

$$
H_{0} Y_{0}=[\underbrace{0 \cdots 0}_{-k} r_{0}]^{t}
$$

with the last row of $Y_{0}$ equal to $I$. Remark that $Y_{0}$ is found by simple solution of a triangular system, which corresponds to finding the first $-k+1$ terms of the formal series for $F(z)^{-1}$.

Back to $k \geqslant 0$ now. (4) gives recursively the denominators in $D_{k}$, but we could use the same recursion coefficients for the computation of the residual columns. This follows e.g. from (5). The recursion coefficients in (4) only depend upon the diagonal and lower diagonal of $\widetilde{L}_{N} D_{N}$, defined in (5). Thus, it seems more natural to compute the columns of $\widetilde{L}_{N} D_{N}$ than to compute the columns of $U_{N}$. This corresponds to a continued fraction approach [4]. The recursion follows directly from (4) and (5) and can be found e.g. in [13]. It is not different from the scalar case [5], [6]. We in fact then compute the RCF

$$
p^{[k / 0]}+z^{k-1} \frac{\left.u_{1} z^{2}\right]}{\mid I+v_{1} z}+\frac{u_{2} z^{2}}{\mid I+v_{2} z}+\cdots
$$

with

$$
u_{i}=-r_{i-2}^{-1} r_{i-1} \text { and } \quad v_{i}=-r_{i-1}^{-1}\left(r_{i-1}^{\prime}+r_{i-2}^{\prime} u_{i}\right)
$$

There is of course a similar LCF version for the computation of the $L$-numerator and $L$-denominator of the PA.

As an example we give the recursion for the right-hand side $R=\widetilde{L}_{N} D_{N}$ of (5). Call the nonzero part in the $i$ th column of $R R_{i}$ for $i=0,1, \ldots, N$, then $R_{i}$ consists of the $N-i+1$ first rows of a column vector $A_{i}$, having $2(N-i)+1$ elements. The recursion for the columns $A_{i}$ is as follows:

$$
\begin{gathered}
A_{-1}=[\underbrace{0 \cdots 0}_{2 N+2}]^{t}, \quad r_{-1}=I, r_{-1}^{\prime}=0, \\
A_{0}=\left[h_{0} \cdots h_{2 N}\right]^{t}, \quad r_{0}=h_{0}, r_{0}^{\prime}=h_{1},
\end{gathered}
$$

and for $i=0,1, \ldots, N-1$,

$$
\left.\left.\left.\left.A_{i+1}=\right\urcorner(\neg(\lrcorner\lrcorner A_{i-1} u_{i+1}+A_{i}\right)+\right\lrcorner A_{i} v_{i+1}\right)
$$

$u_{i+1}$ and $v_{i+1}$ as in (9) and $r_{i}$ and $r_{i}^{\prime}$ are the first two elements in $A_{i}$. It is an easy exercise to rewrite this algorithm as a "row-by-row" algorithm so that one need not fix $N$ beforehand and that the increase of $N$ to $N+1$ only requires the computation of an additional row. Such an algorithm in the $L$-version can be found in $[13,(2.9)]$.

In the following we will refer to the recursion (4) as an algorithm to compute the upper-triangular factor $U_{N}$ for $H_{N}^{-1}$, where $H_{N}$ is some given Hankel matrix. Similarly, the recursion (10) will be referred to as an algorithm to compute the lowertriangular factor $\widetilde{L}_{N} D_{N}$ of (5) for a given Hankel matrix $H_{N}$. 
3. Descending Staircases. The computation of the staircases

$$
T_{k}=\{[k+j / j],[k+1+j / j]\}_{j=0}^{\infty} \quad \text { if } k \geqslant+0
$$

and

$$
T_{k}=\{[j /-k+j],[j /-k+1+j]\}_{j=0}^{\infty} \text { if } k \leqslant-0, *
$$

which are interleavings of two adjacent diagonals, introduces no novelty with respect to the previous section and the scalar case. The same factorizations as in Section 2 may be obtained for the Hankel matrix $H_{i}$, along with a shifted Hankel matrix $\bar{H}_{i}=$ $T^{[k+i+2 / i]} J(k \geqslant+0)$ or $\bar{H}_{i}=T^{[i+1 /-k+i+1]} J(k \leqslant-0)$. Remark that for $k \geqslant+0$

$$
\bar{H}_{i} \bar{Y}_{i}=\left[\begin{array}{llll}
0 & \cdots & 0 & \bar{r}_{i}
\end{array}\right]^{t} \Rightarrow H_{i} \bar{Y}_{i}=\left[\begin{array}{llll}
\bar{p}_{i} & 0 & \cdots & 0
\end{array}\right]^{t} .
$$

This proves the assertion about $Y_{N}^{(N)}$ given after relation (8).

The recursion (4) now falls apart into two steps alternating between $D_{k}$ and $D_{k \pm 1}$ with $\pm=\operatorname{sign} k$. For more details consult [5]

4. Antidiagonals and Ascending Staircases. With the same techniques as used in Section 2, we see that for the computation of the antidiagonals

$$
E_{k}=\{[k-j / j]\}_{j=0}^{k} \text { for } k \geqslant 0
$$

the solution of the systems

$$
\begin{aligned}
\left(J T^{[k-j / j]}\right) Q^{[k-j / j]} & =\left[0 \cdots 0 p_{k-j}^{[k-j / j]}\right]^{t}, \quad j=0,1, \ldots, k, \\
& \equiv G_{j} Q_{j}=e_{j} p_{j} \quad \text { with } q_{j}^{[k-j / j]}=I,
\end{aligned}
$$

are involved. $G_{j}$ is again a Hankel matrix and system (11) is of the same form as (3). The recursion (4) remains valid with obvious notational transcriptions. The factorization corresponding to (5) now has another Padé interpretation. The upper-triarigular factor in the LHS has $I$ as diagonal elements and contains in its columns the unreversed and monically normalized $R$-denominators, while the lower-triangular matrix in the RHS contains reversed $R$-numerators.

From their definitions (3) and (11) it follows that the following relation exists between the Hankel matrices $G$ and $H^{* *}$

$$
H_{j}^{(k)}=T^{[k+j+1 / j]} J=J\left(J T^{[k+2 j+1-j / j]}\right) J=J G_{j}^{(k+2 j+1)} J .
$$

Thus, relation (8) becomes

$$
G_{i}^{(k+2 i+1)} J X J=J P J,
$$

where $X$ is the lower-triangular and $P$ the upper-triangular of (8). This is a factorization of the form (5) and from the above interpretation it follows that the columns of $X$ are reversed $R$-denominators of $D_{k}$ and that the columns of $P$ are the corresponding $R$-numerators.

* A path with negative index in the Padé table is the reflection in the main diagonal of the corresponding positively indexed path. Since the reflection of $T_{0}$ is not $T_{0}$ any more, we distinguish between $T_{+0}$ and $T_{-0}$ as above.

** We use a superscript between brackets to indicate the diagonal that is involved. 
The diagonals

$$
E_{k}=\{[j /-j-k]\}_{j=0}^{k} \text { for } k<0
$$

are nothing else but the $E_{-k}$ diagonals in reversed order. The elements of these diagonals can be found by applying the same computations as for the $E_{-k}$ diagonals on the $F(z)^{-1}$ series.

Remark that the duality emanating from the algorithms (4) and (10) for $D_{k}$, computing the triangular factor in the LHS (denominators) resp. RHS (residuals) of (5), becomes, when using similar algorithms for $E_{k}$, a duality between denominators (LHS) and numerators (RHS). The algorithm (4) for $E_{-k}(k>0)$ using the $F(z)^{-1}$ series thus computes the RHS triangular factor of (5) from right to left, instead of from left to right as algorithm (10) does when computing $E_{k}(k>0)$ using the $F(z)$ series. The matrix interpretation confirms this result. Note that $G_{k}=J T^{[0 / k]}$, with $T^{[0 / k]}$ a lower-triangular Toeplitz matrix, based on the coefficients of the series $F(z)$. The inverse of $T^{[0 / k]}$ is again lower-triangular Toeplitz, based on the coefficients in the series $F(z)^{-1}$, i.e.

$$
\left(T^{[0 / k]}(F)\right)^{-1}=T^{[0 / k]}\left(F^{-1}\right)
$$

Thus, if $G_{k}(F) U(F)=L(F)$ with $U$ upper- and $L$ lower-triangular, then

$$
J U(F) J=\left(J\left(G_{k}(F)\right)^{-1} J\right)(J L(F) J) .
$$

Compare this with

$$
L\left(F^{-1}\right)=G_{k}\left(F^{-1}\right) U\left(F^{-1}\right)
$$

then it follows that

$$
J U(F) J=L\left(F^{-1}\right) \text { and } J L(F) J=U\left(F^{-1}\right) .
$$

The ascending staircases, defined as

$$
U_{k}=\left\{\{[k-j / j],[k-1-j / j]\}_{j=0}^{k-1},[0 / k]\right\} \text { for } k \geqslant 0,
$$

and

$$
U_{k}=\left\{\{[j /-k-j],[j /-k-j-1]\}_{j=0}^{k-1},[-k / 0]\right\} \quad \text { for } k<0,
$$

can again be handled in a similar way. Like for the diagonals, the algorithms are directly transcribed from the scalar case [5], [6].

5. Rows, Sawteeth and Related Algorithms. For the row $L_{k}=\{[k / j]\}_{j=0}^{\infty}$ $(k \geqslant 0)$ we must find $R$-denominators from the set of systems

$$
\begin{aligned}
& T^{[k+1 / j]} Q^{[k / j]}=e_{j} r_{j}^{[k / j]} \equiv T_{j} Q_{j}=e_{j} r_{j}, \quad \text { or } \\
& T^{[k / j]} Q^{[k / j]}=e_{0} p_{j}^{[k / j]} \equiv \underline{T}_{j} Q_{j}=e_{0} p_{j} .
\end{aligned}
$$


The following nesting of the Toeplitz matrices is essential

$$
T_{j+1}=\left[\begin{array}{c|c}
t_{0} & W_{j}^{t} \\
\hline V_{j} & T_{j}
\end{array}\right]=\left[\begin{array}{l|l}
T_{j} & \hat{w}_{j} \\
\hline \hat{V}_{j}^{t} & t_{0}
\end{array}\right]
$$

with $t_{i}=f_{k+1+i}$ and $\underline{T}_{j+1}$ similarly with $\underline{t}_{i}=t_{i-1}, i=0,1, \ldots$, instead of $t_{i}$.

The recursion for the $R$-denominators are [5] (monic normalization)

$$
Q_{j+1}=\left[\begin{array}{c}
0 \\
Q_{j}
\end{array}\right]-\left[\begin{array}{c}
0 \\
Q_{j-1} \\
0
\end{array}\right] p_{j-1}^{-1} p_{j}+\left[\begin{array}{c}
Q_{j} \\
0
\end{array}\right]_{j}^{-1} r_{j-1} p_{j-1}^{-1} p_{j}
$$

with

$$
\left[\begin{array}{l}
p_{j} \\
r_{j}
\end{array}\right]=\left[\begin{array}{c}
W_{j}^{t} \\
\hat{V}_{j}^{t}
\end{array}\right] Q_{j}
$$

and initial conditions

$$
\begin{array}{rlrl}
Q_{-1} & =[\phi], & p_{-1}=I, & r_{-1}=-I, \\
Q_{0}=I, & p_{0}=t_{-1}, & r_{0}=t_{0}
\end{array}
$$

The matrix factorization interpretation is the following

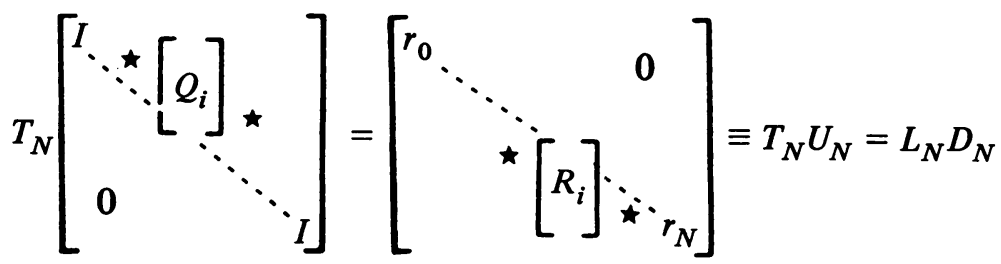

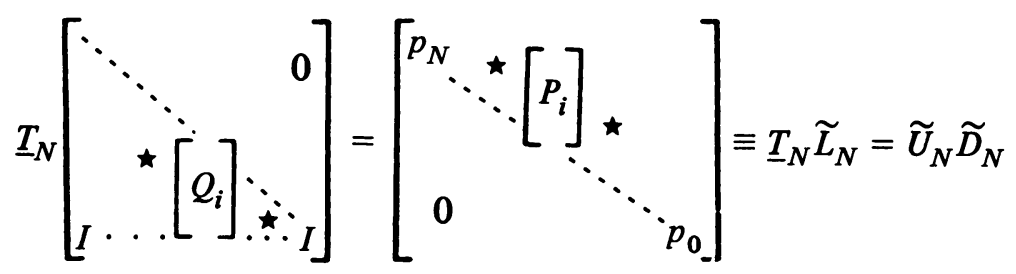

with $\widetilde{D}_{N}=\operatorname{diag}\left(p_{N} \cdots p_{0}\right) . \quad U_{N}$ and $\widetilde{L}_{N}$ contain the $R$-denominators, $L_{N} D_{N}$ contains part of the corresponding $R$-residuals (the lowest degree nonzero coefficients) and $\widetilde{U}_{N} \widetilde{D}_{N}$ (part of) the corresponding $R$-numerators. The recursion coefficients in (14) are constructed from the diagonal elements in $D_{N}$ and $\widetilde{D}_{N}$. 
The corresponding $L$-version of (15) is

$$
{ }_{N} L_{N} T={ }_{N} D_{N} U \text { and }{ }_{N} \widetilde{U}_{N} \underline{T}={ }_{N} \widetilde{D}_{N} \widetilde{L}^{* * *}
$$

with ${ }_{N} T=T_{N}^{t},{ }_{N} \underline{T}=\underline{T}_{N}^{t} \cdot{ }_{N} U$ and ${ }_{N} \widetilde{U}$ are upper-triangular and ${ }_{N} L$ and ${ }_{N} \widetilde{L}$ are lower-triangular. We have

$$
T_{N}=L_{N} D_{N} U_{N}^{-1} \quad \text { and } \quad{ }_{N} T=T_{N}^{t}={ }_{N} L^{-1}{ }_{N} D_{N} U
$$

Instead of the $L D U$ factorization of $T_{N}$ and ${ }_{N} T$ as given in (16), we could also look for an $U D L$ factorization, viz.

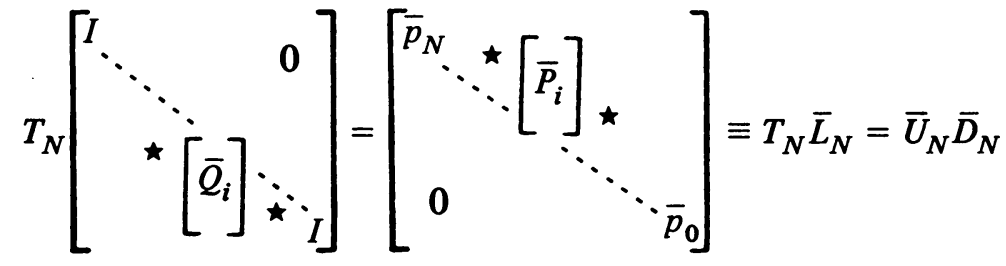

with $\bar{D}_{N}=\operatorname{diag}\left(\bar{p}_{N} \cdots \bar{p}_{0}\right)$

From the second factorization in (15) we see that the $\bar{Q}_{i}$ are $R$-denominators for row $L_{k+1}$ with a comonic normalization and $\bar{P}_{i}$ the corresponding $R$-numerators. A similar $L$-dual is

$$
{ }_{N} \bar{U}_{N} T={ }_{N} \bar{D}_{N} \bar{L}
$$

Comparing (15), (16), (17) and (18) and using $J T_{N}^{t} J=T_{N}$, we get

$$
\begin{aligned}
T_{N} & =L_{N} D_{N} U_{N}^{-1}=\bar{U}_{N} \bar{D}_{N} \bar{L}_{N}^{-1} \\
& =\left(J_{N} \bar{U}^{-1} J\right)\left(J_{N} \bar{D} J\right)\left(J_{N} \bar{L} J\right)=\left(J_{N} L^{-1} J\right)\left(J_{N} D J\right)\left(J_{N} U J\right),
\end{aligned}
$$

from which we conclude that doing the $L D U$ factorization of $T_{N}$ requires in fact the same computations as the $U D L$ factorization of $T_{N}^{t}$ and conversely.

Note also e.g. that $L_{N}=J_{N} \bar{U}^{-1} J$, where $L_{N}$ contains the $R$-residuals for $L_{k}$ as columns and ${ }_{N} \bar{U}$ contains reversed $L$-denominators for $L_{k+1}$ as rows, etc.

With the notations already introduced we have

(20a)

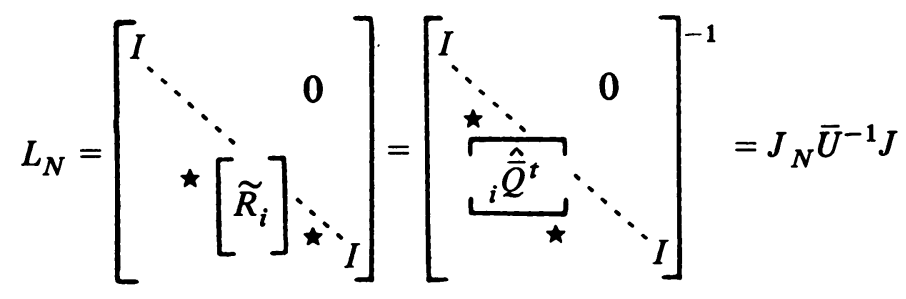

*** The graphical representation is derived from (15) by taking the block transpose, using the rules for the ordinary transpose and transferring the indices from right to left. 
with $\widetilde{R_{i}}=R_{i} r_{i}^{-1}$ (normalized residuals) and

(20b)

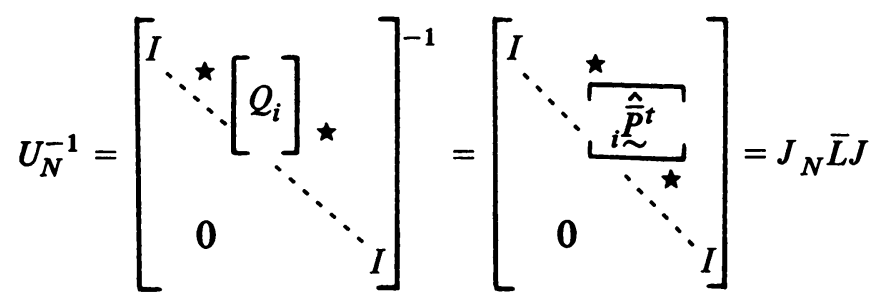

with $\bar{P}_{i} \bar{P}^{t}={ }_{i} \bar{p}^{-1}{ }_{i} \bar{P}^{t}$ (normalized numerators) and

$$
D_{N}=\operatorname{diag}\left(r_{0} \cdots r_{N}\right)=\operatorname{diag}\left({ }_{0} \bar{p} \cdots{ }_{N} \bar{p}\right)=J_{N} \bar{D} J
$$

etc.

The factorizations of $T_{N}$ and $T_{N}^{-1}$ then are

(21a)

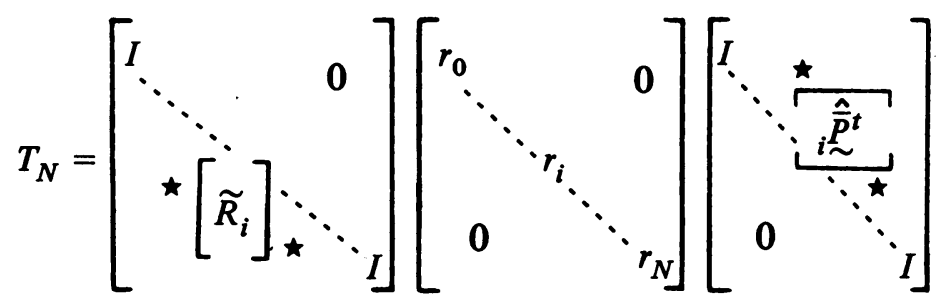

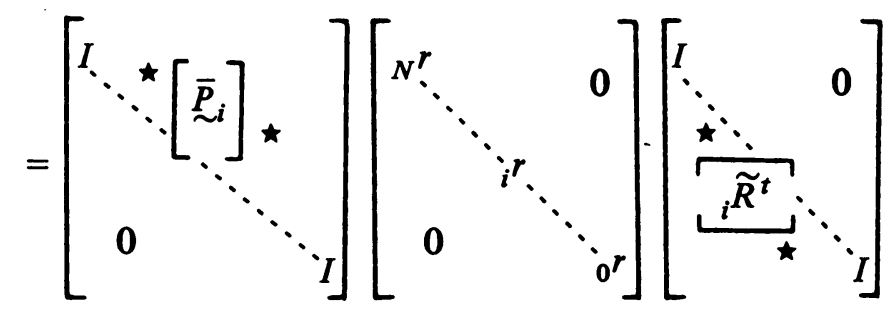

and

(21b)

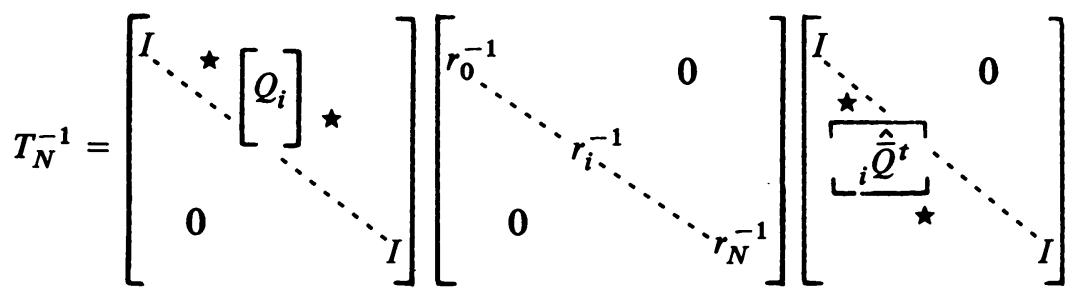

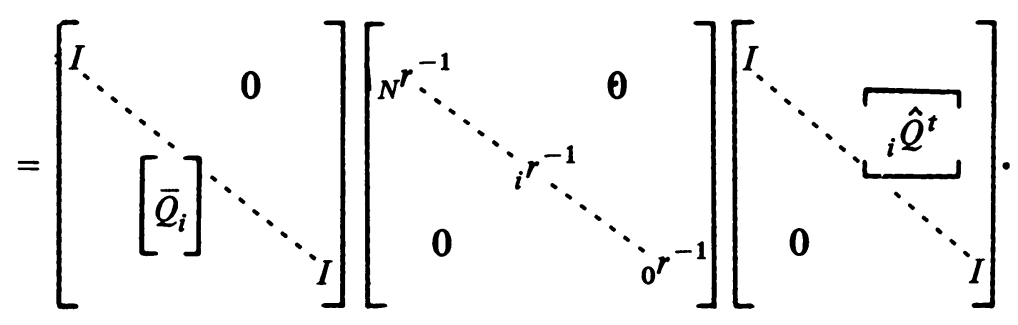

Also,

$$
\left(J_{N} \bar{U} J\right) T_{N} U_{N}=\operatorname{diag}\left(r_{0} \cdots r_{N}\right)
$$


which expresses the biorthogonality of $\left\{{ }_{i} \hat{\bar{Q}}\right\}_{0}^{N}$ and $\left\{Q_{i}\right\}_{0}^{N}$ w.r.t. $T_{N}[2]$.

We are now able to derive a lot of alternative algorithms for (14). The recursion coefficients of (14) depend on the diagonal elements in $D_{N}$ and $\widetilde{D}_{N}$ (see (15)). In (14) they were found as inner products. We could now replace one or two of the inner products by recursions for the columns of $L_{N} D_{N}$ or of $\widetilde{U}_{N} \widetilde{D}_{N}$. The way this is done is trivial and in the style of the derivation of (10). Computing both $L_{N} D_{N}$ (residuals) and $\widetilde{U}_{N} \widetilde{D}_{N}$ (numerators) by recursion makes the explicit evaluation of $U_{N}$, or what is the same $\widetilde{L}_{N}$ (both contain the same denominators), superfluous. This would be a continued fraction algorithm as given in [4].

If we want to compute two adjacent rows $L_{k}$ and $L_{k+1}$, then we have several possibilities like sawtooth variants, etc. [6]. The most elegant is probably (see also, $[1],[13],[15],[18])$

$$
\begin{aligned}
& Q_{i+1}=\left[\begin{array}{l}
0 \\
Q_{i}
\end{array}\right]-\left[\begin{array}{l}
\bar{Q}_{i} \\
0
\end{array}\right] \alpha_{i} \quad \text { with } \alpha_{i}=\bar{p}_{i}^{-1} p_{i}, \\
& \bar{Q}_{i+1}=\left[\begin{array}{l}
\bar{Q}_{i} \\
0
\end{array}\right]-\left[\begin{array}{l}
0 \\
Q_{i}
\end{array}\right] \beta_{i} \quad \text { with } \beta_{i}=r_{i}^{-1} \bar{r}_{i} \text { and with } \\
& {\left[\begin{array}{l}
p_{i} \\
r_{i}
\end{array}\right]=\left[\begin{array}{l}
W_{i}^{t} \\
\hat{V}_{i}^{t}
\end{array}\right] Q_{i} \text { and }\left[\begin{array}{l}
\bar{p}_{i} \\
\bar{r}_{i}
\end{array}\right]=\left[\begin{array}{l}
\bar{W}_{i}^{t} \\
\hat{V}_{i}^{t}
\end{array}\right] \bar{Q}_{i} .}
\end{aligned}
$$

$V_{i}, W_{i}$ and $\underline{V}_{i}$ have been defined earlier and $\bar{w}_{i}^{t}=\left[t_{0} \cdots t_{-i}\right], Q_{i}$ is a monically normalized denominator for $L_{k}$ and $\bar{Q}_{i}$ is a comonically normalized denominator for $L_{k+1}$. In the scalar case is $r_{i}=\bar{p}_{i}$ (see (20)) and this coefficient can be found by recursion, viz.

$$
r_{i+1}=r_{i}-\bar{r}_{i} \bar{p}_{i}^{-1} p_{i}=r_{i}\left(I-\beta_{i} \alpha_{i}\right)
$$

(23b) remains true in the matrix case, but the recursion for $\bar{p}_{i}$ becomes

$$
\bar{p}_{i+1}=\bar{p}_{i}-p_{i} r_{i}^{-1} \bar{r}_{i}=\bar{p}_{i}\left(I-\alpha_{i} \beta_{i}\right) \text {. }
$$

Because ${ }_{i} \bar{p}=r_{i}$ and $\bar{p}_{i}={ }_{i} r, i=0,1, \ldots$, it only requires little effort to find simultaneously the other triangular factors of $T_{N}^{-1}$, containing the $L$-denominators ${ }_{i} Q$ and ${ }_{i} \bar{Q}$. Indeed, (23) becomes in its $L$-version

$$
\begin{aligned}
{ }_{i+1} Q^{t} & =\left[0{ }_{i} Q^{t}\right]-{ }_{i} \alpha\left[{ }_{i} \bar{Q}^{t} 0\right], \quad{ }_{i} \alpha={ }_{i} p_{i} \bar{p}^{-1}, \\
{ }_{i+1} \bar{Q}^{t} & =\left[{ }_{i} \bar{Q}^{t} 0\right]-{ }_{i} \beta\left[0{ }_{i} Q^{t}\right], \quad{ }_{i} \beta={ }_{i} \bar{r}_{i} r^{-1}, \\
{ }_{i} \bar{p} & =r_{i}, \quad{ }_{i} p={ }_{i} Q^{t} W_{i}, \\
{ }_{i} r & =\bar{p}_{i}, \quad{ }_{i} \bar{r}={ }_{i} \bar{Q}^{t} \hat{V}_{i},
\end{aligned}
$$


and also

$$
{ }_{i+1} r={ }_{i} r-{ }_{i} p_{i} \bar{p}^{-1}{ }_{i} \bar{r}=\left(I-{ }_{i} \alpha{ }_{i} \beta\right){ }_{i} r \text {. }
$$

Some further simplification is possible. Using the persymmetry of ${ }_{N} T^{-1}$, it is possible to find that (see [1]) ${ }_{i} p=p_{i}$ and ${ }_{i} \bar{r}=\bar{r}_{i}$. So that (23) and $\left(23^{t}\right)$ can be summarized in the following scheme that is to be executed in parallel.

\section{Initialization}

$$
\begin{aligned}
& \begin{array}{l|l}
r_{0}=t_{0} & { }_{0} r=t_{0} \\
Q_{0}=\bar{Q}_{0}=I & { }_{0} Q={ }_{0} \bar{Q}=I
\end{array} \\
& \text { for } i=0,1,2, \ldots \\
& p_{i}=\left[t_{-i} \cdots t_{i-1}\right] Q_{i}={ }_{i} p={ }_{i} Q^{t}\left[t_{-1} \cdots t_{-i-1}\right]^{t} \\
& \bar{r}_{i}=\left[t_{i+1} \cdots t_{1}\right] \bar{Q}_{i}={ }_{i} \bar{r}={ }_{i} \bar{Q}^{t}\left[t_{i+1} \cdots t_{1}\right]^{t} \\
& \alpha_{i}={ }_{i}{ }^{-1} p_{i} \\
& \beta_{i}=r_{i}^{-1} \bar{r}_{i} \\
& Q_{i+1}=\left[\begin{array}{l}
0 \\
Q_{i}
\end{array}\right]-\left[\begin{array}{c}
\bar{Q}_{i} \\
0
\end{array}\right] \alpha_{i} \\
& { }_{i} \alpha={ }_{i} p r_{i}^{-1} \\
& { }_{i} \beta={ }_{i}{ }_{i}{ }^{-1} \\
& \bar{Q}_{i+1}=\left[\begin{array}{c}
\bar{Q}_{i} \\
0
\end{array}\right]-\left[\begin{array}{c}
0 \\
Q_{i}
\end{array}\right] \beta_{i} \\
& r_{i+1}=r_{i}\left(I-\beta_{i} \alpha_{i}\right) \\
& =\left(I-{ }_{i} \beta{ }_{i} \alpha\right) r_{i} \\
& \begin{aligned}
{ }_{i+1} Q^{t} & =\left[\begin{array}{c}
0 \\
{ }_{i} Q
\end{array}\right]^{t}-{ }_{i} \alpha\left[\begin{array}{c}
{ }_{i} \bar{Q} \\
0
\end{array}\right]^{t} \\
i+1 & \bar{Q}^{t}=\left[\begin{array}{c}
{ }_{i} \bar{Q} \\
0
\end{array}\right]^{t}-{ }_{i} \beta\left[\begin{array}{c}
0 \\
{ }_{i} Q
\end{array}\right]^{t} \\
i+1 & =\left(I-{ }_{i} \alpha{ }_{i} \beta\right)_{i} r \\
& ={ }_{i} r\left(I-\alpha_{i} \beta_{i}\right)
\end{aligned}
\end{aligned}
$$

This scheme computes the triangular factors of $T_{N}^{-1}$ and ${ }_{N} T^{-1}$; see (21). It is like the algorithm in [1] and is a generalization of the block Levinson algorithm [11], [17] which computes row $L_{0}$ in a Laurent-Padé table [10] and in that case also reduces to the recursion for the Szegö orthogonal polynomials [14], [9], [7]. A continued fraction-like approach [4] gives the extension of the ladder-form analog [12] of the Levinson algorithm, and this computes the triangular factors of $T_{N}$ and ${ }_{N} T$ themselves.

A derivation of the algorithm can be found in [13] e.g. We will do the work over again because it will give a better insight in what is happening in Padé terms.

Set $A_{N}=L_{N} D_{N}$ with $L_{N} D_{N}$ as in (15). Thus, $A_{N}$ contains $R$-residuals; and if we call its elements $a_{i j}$, then

$$
T_{N} U_{N}=\left[\begin{array}{cccccc}
a_{00} & & & & \\
a_{10} & a_{11} & & & \\
\vdots & \vdots & \ddots & 0 & \\
\vdots & \vdots & \ddots & \ddots & \\
a_{N 0} & a_{N 1} & & & \ddots & a_{N N}
\end{array}\right]
$$


with

$$
a_{i j}=\left[t_{i} \cdots t_{0}\right]\left[Q_{j}^{t} 0 \cdots 0\right]^{t}=r_{i-j}^{[k / j]}, \quad 0 \leqslant j \leqslant i .
$$

Using the previous scheme to compute $Q_{j}$, we obtain $a_{i 0}=t_{i}, i=0,1, \ldots$, and recursively for $i>0$,

$$
\begin{aligned}
& a_{i, j+1}=\left[t_{i} \cdots t_{0}\right]\left[Q_{j+1}^{t} 0 \cdots 0\right]^{t}
\end{aligned}
$$

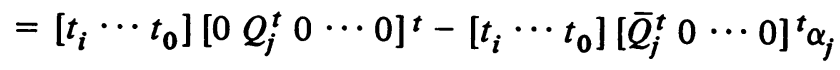

$$
\begin{aligned}
& =a_{i-1, j}-\bar{a}_{i-1, j} \alpha_{j} \text {. }
\end{aligned}
$$

Thus, $\bar{a}_{i-1, j}=\left[t_{i} \cdots t_{0}\right]\left[\bar{Q}_{j}^{t} 0 \cdots 0\right]^{t}$ is the $(k+1+i)$ th coefficient in the $R$ residual that corresponds to $\bar{Q}_{j}$ thus $\bar{a}_{i-1, j}=r_{i-1-j}^{[k+1 / j]}$.

The recursion for $\bar{a}_{i j}$ is similar

$$
\bar{a}_{i 0}=t_{i+1}
$$

and

$$
\begin{aligned}
& \bar{a}_{i, j+1}=\left[t_{i+1} \cdots t_{0}\right]\left[\begin{array}{lll}
\bar{Q}_{j+1}^{t} & 0 \cdots 0
\end{array}\right]^{t}
\end{aligned}
$$

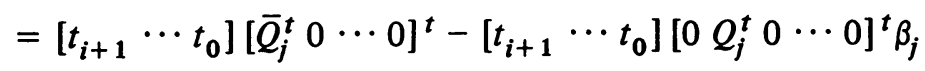

$$
\begin{aligned}
& =\bar{a}_{i j}-a_{i j} \beta_{j} \text {. }
\end{aligned}
$$

Clearly, $\bar{r}_{j}=r_{0}^{[k+1 / j]}=\bar{a}_{i j}$ and $r_{j}=r_{0}^{[k / j]}=a_{j j}$

From (24) we obtain the $\bar{r}_{j}$ and $r_{j}$, but to find the other two coefficients $p_{i}={ }_{i} p$ and $\bar{p}_{i}={ }_{i} r$, needed to compute $\alpha_{i}, \beta_{i},{ }_{i} \alpha$ and ${ }_{i} \beta$, we have to do another factorization. Indeed, the coefficients $p_{i}$ and $\bar{p}_{i}$ are found from the factorization (17), i.e.

$$
T_{N} \bar{L}_{N}=\bar{B}_{N} \quad\left(=\bar{U}_{N} \bar{D}_{N}\right)
$$

$\bar{B}_{N}$ contains the $R$-numerators for row $k+1$. We number the elements of $\bar{B}_{N}$ in reverse order, so that

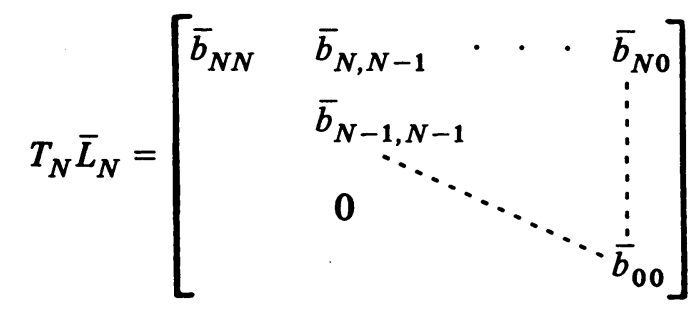

with

$$
\bar{b}_{i j}=\left[\begin{array}{lll}
t_{0} & \cdots & t_{-i}
\end{array}\right]\left[\begin{array}{llll}
0 & \cdots & 0 & \bar{Q}_{j}^{t}
\end{array}\right]^{t}=p_{k+1-(i-j)}^{[k+1 / j]}, \quad 0 \leqslant j \leqslant i .
$$

Using again the recursion for $\bar{Q}_{j}$, we get

$$
\bar{b}_{i 0}=t_{-i}, \quad i=0,1,2, \ldots,
$$


and

$$
\begin{aligned}
& \bar{b}_{i, j+1}=\left[t_{0} \cdots t_{-i}\right]\left[\begin{array}{llll}
0 & \cdots & 0 & \bar{Q}_{j+1}^{t}
\end{array}\right]^{t}
\end{aligned}
$$

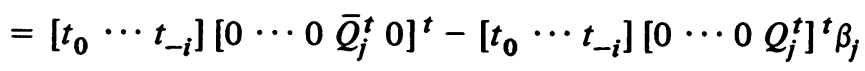

$$
\begin{aligned}
& =\bar{b}_{i-1, j}-b_{i-1, j} \beta_{j}
\end{aligned}
$$

with $b_{i j}=\left[\begin{array}{lll}t_{0} & \cdots & t_{-i-1}\end{array}\right]\left[\begin{array}{llll}0 & \cdots & 0 & Q_{j}^{t}\end{array}\right]^{t}=p_{k-(i-j)}^{[k / j]}$. Thus, $b_{i j}$ are $R$-numerator coefficients for the $k$ th row $L_{k}$ of the Padé table. They can be found by the recursion:

$$
b_{i 0}=t_{-i-1}
$$

and

$$
\begin{aligned}
& b_{i, j+1}=\left[t_{0} \cdots t_{-i-1}\right]\left[\begin{array}{llll}
0 & \cdots & 0 & Q_{j+1}^{t}
\end{array}\right]^{t}
\end{aligned}
$$

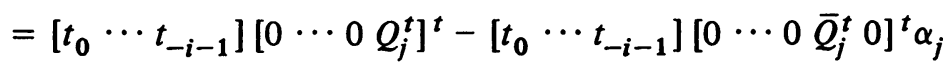

$$
\begin{aligned}
& =b_{i j}-\bar{b}_{i j} \alpha_{j} \text {, }
\end{aligned}
$$

and $p_{i}={ }_{i} p=b_{i i}$ and $\bar{p}_{i}={ }_{i} r=\bar{b}_{i i}$.

(24) and (25) put together give an algorithm to find the RCF coefficients of the PA's that are in row $L_{k}$ without any inner product evaluation. The scheme is summarized as

$$
\begin{aligned}
& a_{00}=t_{0} \\
& \bar{a}_{00}=t_{1}
\end{aligned}
$$

$$
\mid \begin{aligned}
& \bar{b}_{00}=t_{0} \\
& b_{00}=t_{-1}
\end{aligned}
$$

$$
\text { for } i=0,1,2, \ldots
$$

$$
\begin{aligned}
& \beta_{i}=a_{i i}^{-1} \bar{a}_{i i} \\
& a_{i+1,0}=t_{i+1} \\
& \bar{a}_{i+1,0}=t_{i+2}
\end{aligned}
$$$$
\mid \begin{aligned}
& \alpha_{i}=\bar{b}_{i i}^{-1} b_{i i} \\
& \bar{b}_{i+1,0}=t_{-i-1} \\
& b_{i+1,0}=t_{-i-2}
\end{aligned}
$$

$$
\text { for } j=1,2, \ldots, i+1
$$

$$
\begin{array}{l|l}
a_{i+1, j}=a_{i, j-1}-\bar{a}_{i, j-1} \alpha_{j-1} & \bar{b}_{i+1, j}=\bar{b}_{i, j-1}-b_{i, j-1} \beta_{j-1} \\
\bar{a}_{i+1, j}=\bar{a}_{i+1, j-1}-a_{i+1, j-1} \beta_{j-1} & b_{i+1, j}=b_{i+1, j-1}-\bar{b}_{i+1, j-1} \alpha_{j-1}
\end{array}
$$

Herein we compute the left triangular factors in the $U L$ factorization of $T_{N}=T^{[k+1 / N]}$ and $\bar{T}_{N}=T^{[k+2 / N]}$ and the left triangular factors in the $L U$ decomposition of $T_{N}$ and $\underline{T}_{N}=T^{[k / N]}$. The right triangular factors in these decompositions are obtained when using a similar scheme for the left residuals and numerators.

As before, we can apply the algorithm on the series $F(z)^{-1}$ to obtain recursions for columns or vertical sawteeth in the Padé table. 
6. Conclusion. Via a matrix factorization interpretation of recursive matrixPadé algorithms, there is no problem to carry over all the algorithms from the scalar case, provided the matrix-Padé table is normal.

Some variants of the algorithms given by Akaike [1] and Rissanen [13] for the factorization of Toeplitz matrices are given a Padé interpretation. In this way we obtain generalizations of the Levinson-Wiggins-Robinson [11], [17] algorithm and the so-called ladder form algorithm [12] for linear prediction.

Afdeling Toegepaste Wiskunde en Programmatie

K. U. Leuven

Celestijnenlaan 200A

B-3030 Heverlee, Belgium

1. H. AKAIKE, “Block Toeplitz matrix inversion," SIAM J. Appl. Math., v. 24, 1973, pp. 234-241.

2. G. BAXTER, "Polynomials defined by a difference system," J. Math. Anal. Appl., v. 2, 1961 , pp. 223-263.

3. D. BESSIS, "Topics in the theory of Padé approximants," in Padé Approximants (P. R. Graves-Morris, Ed.), The institute of Physics, London-Bristol, 1973, pp. 19-44.

4. A. BULTHEEL, "Division algorithms for continued fractions and the Pade table," J. Comput. Appl. Math. (To appear.)

5. A. BULTHEEL, "Fast factorization algorithms and Padé approximation: a survey." (Submitted.)

6. A. BULTHEEL, "Recursive algorithms for the Padé table: Two approaches," in Padé Approximation and its Applications (L. Wuytack, Ed.), Lecture Notes in Math., vol. 765, SpringerVerlag, Berlin, 1979, pp. $211-230$.

7. P. DELSARTE, Y. GENIN \& Y. KAMP, "Orthogonal polynomial matrices on the unit circle," IEEE Trans. Circuits and Systems, CAS-25, 1978, pp. 149-160.

8. B. W. DICKINSON, M. MORF \& T. KAILATH, "A minimal realization algorithm for matrix sequences," IEEE Trans. Automatic Control, AC-10, 1974, pp. 31-38.

9. L. Y. GERONIMUS, Orthogonal Polynomials, Consultants Bureau, New York, 1961.

10. W. B. GRAGG, "Laurent, Fourier and Chebyshev Padé tables," in Padé and Rational Approximation, Theory and Applications (E. B. Saff and R. S. Varga, Eds.), Academic Press, New York, 1977, pp. 61-72.

11. N. LEVINSON, "The Wiener RMS (root mean square) error criterion in filter design and prediction," J. Mathematical Phys., v. 25, 1947, pp. 261-278.

12. A. MORF, A. VIEIRA \& D. T. LEE, Ladder Forms for Identification and Speech Pro cessing, Proc. Conf. on Decision and Control, New Orleans, Louisiana, Dec. 7-9, 1977.

13. J. RISSANEN, "Algorithms for triangular decomposition of block Hankel and Toeplitz matrices with application to factoring positive matrix polynomials," Math. Comp., v. 27, 1973, pp. 147-154.

14. G. SZEGÖ, Orthogonal Polynomials, Amer. Math. Soc. Colloq. Publ., vol. 23, Amer. Math. Soc., Providence, R. I., 1939.

15. W. F. TRENCH, "An algorithm for the inversion of finite Toeplitz matrices," SIAM J. Appl. Math., v. 12, 1964, pp. 515-522.

16. W. F. TRENCH, "An algorithm for the inversion of finite Hankel matrices," SIAM $J$. Appl. Math., v. 13, 1965, pp. 1102-1107.

17. R. A. WIGGINS \& E. A. ROBINSON, "Recursive solution to the multichannel filtering problem," J. Geophys. Res., v. 70, 1965, pp. 1885-1891.

18. S. ZOHAR, "Toeplitz matrix inversion. The algorithm of W. F. Trench," J. Assoc. Comput. Mach., v. 16, 1969, pp. 592-601. 